\title{
Asymmetric Divisions, Aggresomes and Apoptosis
}

\author{
Aakanksha Singhvi ${ }^{*}$ and Gian Garriga \\ Molecular and Cell Biology, University of California, Berkeley, CA 94720-3204, USA
}

\begin{abstract}
Asymmetric cell division (ACD) is a fundamental process used to generate cell diversity during metazoan development and occurs when a cell divides to generate daughter cells that adopt distinct fates(Horvitz and Herskowitz, 1992; Knoblich, 2001). Stem cell division is also a type of ACD and provides a source of new cells during development and in adult animals. Some ACDs produce a daughter cell that dies. The logic of why a cell divides to generate a dying daughter remains elusive. Recently it was shown that denatured proteins are segregated asymmetrically during cell division, We review recent data that provides interesting insights into how apoptosis is regulated during ACD and speculate on potential connections between ACD-induced cell death and partitioning of denatured proteins. We discuss potential mechanisms for this link and what it may imply for development and disease in metazoans.
\end{abstract}

\section{Introduction}

Asymmetric cell division (ACD) is a division that produces daughter cells that adopt distinct fates. Stem cell divisions, for example, are a type of ACD because they produce another stem cell and a cell with more restricted developmental potential. ACD requires the precise coordination of various events. The dividing cell first polarizes along a given axis. This is achieved by the asymmetric distribution of intrinsic molecules, by asymmetric signaling from neighboring cells or by both. This polarization then leads to the asymmetric distribution of cell fate determinants or intracellular signaling components. The mitotic spindle also orients along this axis of polarity to ensure that the determinants or signaling components are inherited by one of the daughter cells(Knoblich, 2001).

An example of intrinsic molecules that regulate cell fate and orientation of the mitotic spindle are the proteins of the Par complex. The first identified components of the complex, PAR-3 and PAR-6, were shown to be necessary for the asymmetric division of the C. elegans zygote and along with aPKC regulate ACD in worms and flies and the polarity of epithelial cells and neurons(Goldstein and Macara, 2007). Through protein interactions, the Par complex can orient the spindle and helps segregate determinants that specify the fate of the daughter cell that inherits them. In Drosophila neuroblasts, for example, the PAR proteins regulate the asymmetric distribution of Prospero, a transcription factor that specifies the fate of one of the daughter cells (REF). Cell signaling can also regulate ACD. In C. elegans secreted Wnt glycoproteins influence many ACDs(Mizumoto and Sawa, 2007). For example, at the fourcell stage of embryonic development, secretion of the Wnt MOM- 2 from the P2 cell orients the spindle of the neighboring EMS blastomere and specifies the fates of its daughter cells.

However, it is not only fate determinants that are segregated asymmetrically during cell division. A recent study shows that aggresomes are asymmetrically segregated during mitosis (Rujano et al., 2006). Aggresomes are microtubule based inclusion bodies that contain

\footnotetext{
* present address: Laboratory of Developmental Genetics, Rockefeller University, New York, NY 10021, USA
} 
misfolded protein aggregates ensheathed in intermediate filaments(Kopito, 2000). Aggresomes form when cells accumulate misfolded proteins that exceed the ability of the proteasome to degrade them: for example, during stress or diseased states(Kopito, 2000). The generation of aggresomes begins when protein aggregates are transported to the microtubule organizing center (MTOC) by dynein-dependent transport along microtubules(Johnston et al., 2002), where they associate with proteosomes and several chaperone proteins, including those of the Hsp40/70 family(Johnston et al., 1998). Protein aggregates at the MTOC can then be degraded by proteosomes or removed by autophagy(Garcia-Mata et al., 2002). In extreme cases, aggresomes can cause mitotic spindle disassembly and apoptosis(Hut et al., 2005; Vidair et al., 1993).

\section{Aggresomes and asymmetric cell division}

To investigate the behavior of aggresomes in cycling cells, a recent study expressed a polyglutamine-containing fragment of the Huntingtin protein in HEK293 and O23 cell culture lines(Rujano et al., 2006). This expression induced the formation of protein aggregates, similar to the aggregates seen in Huntington's disease patients. When followed through cell divisions, aggresomes tend to asymmetrically segregate into only one of the two daughter cells. Cells that inherit the aggresomes were more likely to die (Figure 1A).

Asking whether aggregated proteins are asymmetrically inherited in polarized living tissue, the investigators studied human intestinal crypts of patient with spinocerebellar ataxia 3 , a neurodegenerative disease associated with the accumulation of ataxin-3 polyglutaminecontaining protein. Protein aggregates were rarely found in intestinal stem cells but were present in recycling, short-lived differentiated cell populations, indicating that the asymmetric segregation of aggresomes is not an artifact of cultured cells. The segregation of aggresomes to the short-lived daughters suggests that normal intestinal stem cells segregate aggresomes and that this segregation maintains a healthy population of stem cells may have an important function (Figure 1B).

The ability to asymmetrically segregate aggresomes during mitosis appears to be conserved. Embryonic Drosophila neuroblasts divide in a stem cell pattern to generate another neuroblast and a neural precursor, the ganglion mother cell (GMC)(Betschinger and Knoblich, 2004; Jan and Jan, 2001). The GMC will divide to produce two neurons or glia, and the neuroblast undergoes apoptosis after a limited number of divisions. When a Huntington protein fragment was expressed in the neuroblast lineage, protein aggregates formed and associated with the centrosome that was inherited by the neuroblast, such that aggregates were inherited by the neuroblast(Rujano et al., 2006) (Figure 1C). The authors argued that this pattern of inheritance protected the longer-lived neurons and glia from the potential deleterious effects of protein aggregates.

How might aggresomes be asymmetrically inherited in these ACDs? One clue comes from their sub-cellular distribution. When misfolded proteins accumulate, they form aggregates that are transported to the MTOC by dynein and interact with the peri-centriolar material (PCM), a region that concentrates chaperones and proteosomes when aggregated proteins accumulate (Garcia-Mata et al., 2002) The PCM is also asymmetrically segregated during mitosis(Stearns, 2001), suggesting that the asymmetric segregation of PCM proteins and aggresomes might be linked. Each mitotic spindle has two centrosomes, a mother and a daughter centrosome, that are structurally distinct(Delattre and Gonczy, 2004; Urbani and Stearns, 1999). Before its duplication, the centrosome contains a centriole and PCM(Stearns, 2001). During mitosis, the PCM attaches preferentially to the older or mother centriole, which maintains its astral microtubules. The daughter centrosome, on the other hand, lacks a defined PCM. After the centrosomes occupy position on opposite sides of the nucleus, the daughter centrosome 
nucleates a new PCM de novo(Rebollo et al., 2007; Rusan and Peifer, 2007; Yamashita et al., 2007).

If PCM proteins regulate aggresome distribution, aggresomes should associate with the mother centrosome. Two observations are consistent with this hypothesis. Modified forms of the signaling proteins SMAD1 and $\beta$-catenin proteins that are destined for degradation are targeted to the PCM, ensuring that they are inherited by one of the daughters. This targeting can occur in divisions of human embryonic stem cells, of COS7 cells and of the Drosophila blastoderm, divisions that were thought to be symmetric(Fuentealba et al., 2008). While it seems likely that these proteins are associated with aggresomes, this association has not been investigated. It is also unclear whether this segregation has an important regulatory function for the signaling pathways in which the proteins participate. Also consistent with this hypothesis, dividing Drosophila larval neuroblasts segregate the PCM/mother centrosome to the neuroblast rather than the neural precursor (Rebollo et al., 2007; Rusan and Peifer, 2007). The studies on aggresome segregation focused on embryonic neuroblasts, while those on PCM segregation were with larval neuroblasts. While both the PCM and aggresomes segregate to the apical neuroblast cell, centrosomal orientation occurs at different times and by distinct mechanisms in these neuroblasts(Rebollo et al., 2007; Rusan and Peifer, 2007). To test this model, segregation of the PCM and aggresomes needs to be examined in the same neuroblast divisions.

In both cultured cell and Drosophila neuroblast divisions, the daughter cell that inherits aggresomes will sometimes die(Rujano et al., 2006). This observation raises the interesting question of how aggresomes inheritance and apoptosis are linked.

\section{ACD and apoptosis}

An intriguing type of ACD is one that produces a daughter cell that dies during development. This type of ACD raises interesting questions. Do these divisions use the same mechanisms to distribute developmental potential as ACDs that generate two surviving cells or does the apoptotic fate require distinct mechamisms of ACD? For example, do ACDs that produce apoptotic daughters use known regulators of ACDs such as PAR proteins or Wnt signaling to specify the apoptotic fate? And finally, why divide to produce a cell fated to die?

\section{Do divisions that generate an apoptotic daughter cell use the same mechanisms as ACDs that generate two surviving cells?}

Although broadly expressed, the two $C$. elegans proteins HAM-1 and PIG-1 have so far been reported to act only in ACDs that produce an apoptotic daughter in C.elegans, suggesting that ACDs involving apoptosis might be unique (Cordes et al., 2006; Frank et al., 2005). Other genes that regulate ACDs that produce an apoptotic daughter, however, do act in other types of ACD. In certain Drosophila divisions, the protein Numb is asymmetrically distributed to one daughter cell. Numb inhibits signaling through the Notch receptor. The daughter cell that does not inherit Numb is receptive to Notch ligands, but the daughter that inherits Numb is not, resulting in daughter cells that adopt distinct fates (REF). Besides regulating many ACDs that produce two surviving daughter cells, Notch and Numb also regulate a GMC division that produces a sertonergic neuron and an apoptotic cell in the Drosophila CNS. Similar to ACDs that produce two surviving cells, Notch promotes the fate of one daughter cell, in this case the apoptotic cell, and Numb antagonizes Notch in the surviving cell(Lundell et al., 2003).

Snail and its paralogs Escargot and Worniu regulate the ACD of Drosophila CNS neuroblasts. In the fly, these paralogs regulate two events. First, they transcriptionally regulate the expression of the apical cortical gene inscuteable. Inscuteable protein, which is localized apically by its ability to bind to the PAR-3 ortholog Bazooka, in turn recruits other molecules 
to the apical cortex. These molecules then orient the mitotic spindle along the apical-basal axis and distribute cell fate determinants to daughter cells. Inscuteable, for example, helps localize the determinant Prospero to the GMC. Second, loss of Snail and its paralogs results in spindle orientation and determinant localization defects that are much more severe than those caused by loss of Inscuteable. Thus, Snail and its paralogs also regulate genes that involved in neuroblast ACD in an Inscuteable-independent manner. The direct transcriptional targets that contribute to spindle positioning and the asymmetric localization of determinants are unknown (Ashraf and Ip, 2001; Cai et al., 2001). Whether Snail and its paralogs regulate fly ACDs that produce apoptotic cells is unknown.

Mammalian Slug, a Snail homolog, acts as an anti-apoptotic factor in hematopoietic progenitors by repressing expression of the proapoptotic factor Puma(Wu et al., 2005). Puma, a BH3-only containing protein, binds and inhibits BCL-2. The C. elegans Snail homolog CES-1 directly links ACD and apoptosis in the NSM lineage(Ellis and Horvitz, 1991; Metzstein and Horvitz, 1999; Thellmann et al., 2003). The NSM neuroblast divides asymmetrically to produce a larger NSM neuron and a smaller apoptotic cell(Hatzold and Conradt, 2008). In other $C$. elegans divisions, the apoptotic daughter is smaller than its sister cell(Cordes et al., 2006; Frank et al., 2005). CES-1 binds to regulatory sequences in the egl- 1 gene and represses its expression, leading to reduction of the pro-apoptotic $\mathrm{BH} 3$-only protein encoded by egl-1. While loss of CES-1 produces no defects in the NSM lineage, a gain-of-function ces- 1 mutation causes the NSM sister to survive (Figure 2) (Ellis and Horvitz, 1991).

Loss of CES-2, an HLF transcription factor, also causes the NSM sister to survive, and loss of CES-1 suppresses this phenotype, resulting in the death of the NSM sister. These and additional observations have led to the model that in the NSM sister, CES-2 transcriptionally represses ces-1(Metzstein et al., 1996; Metzstein and Horvitz, 1999). In the absence of CES-1 protein, the bHLH heterodimer containing HLH-2 and HLH-3 transcriptionally activates egl-1, causing the NSM sister to die (Figure 2). A loss-of-function mutation in ces-2 or a gain-of-function mutation in ces- 1 result in increased expression of CES-1, which competes with HLH-2/HLH-3 for an E-box binding site, disrupting egl- 1 activation and allowing the NSM sister to survive (Figure 2)(Thellmann et al., 2003).

As with other molecules necessary for $C$. elegans ACDs that produce apoptotic daughters (Cordes et al., 2006; Frank et al., 2005), CES-1 not only regulates apoptosis, but also the sizes of the daughter cells(Hatzold and Conradt, 2008). Both loss of CES-2 and a gain-of-function mutation in ces- 1 cause the NSM and its sister to be more equivalent in size. As with the effects of apoptosis, this cell size phenotype displayed by the ces-2 mutant is also suppressed by loss of CES-1. It is not known how CES-1 and CES-2 localize in this lineage or how they regulate daughter cell size or positioning.

The ces-1 $(g f)$ mutation also results in the survival of the I2 neuron's sister, a cell that dies in wild-type animals(Ellis and Horvitz, 1991). No other phenotypes were reported for the ces- 1 or ces-2 mutants. So while it is clear that Snail and its paralogs function in Drosophila ACDs that produce two surviving cells, CES-1 and CES-2 are only known to regulate ACDs that produce an apoptotic daughter.

Like $c e s-2, d n j-11$, which encodes a MIDA1-like protein of the Hsp40 chaperone family, also functions upstream of ces-1 to regulate the orientation of the NSM neuroblast division, the sizes of its daughters and the apoptotic fate of the NSM sister (Figure 2)(Hatzold and Conradt, 2008). MIDA1-like proteins contain DnaJ/Hsp40 and Myb domains, and both of these domains are important for DNJ-11 function. How does DNJ-11 regulate ACD and apoptosis? The family of DnaJ/Hsp40 domain containing proteins, along with Hsp70 proteins bind unfolded polypeptides as they exit the ribosome by interacting with hydrophobic residues(Frydman et 
al., 1994). The hydrophobic residues are normally sequestered in the interior of a protein, and their exposure to the hydrophilic environment of the cytosol can cause aggregation and misfolding. MIDA1/Hsp70 ribosome associating complexes (RAC) prevent this aggregation during nascent polypeptide synthesis and ensure a link between translation and proper protein folding(Hundley et al., 2005; Otto et al., 2005). MIDA1 protein localization requires the Myb domain, and regulation of MIDA1 localization has been implicated in localizing Hsp70 activity to specific sub-cellular compartments(Acebron et al., 2008). If DNJ-11 functions as a RAC chaperone in the NSM lineage, it follows that it must do so with a yet-unidentified Hsp70.

Hsp70 proteins have been previously implicated as regulators of both cell proliferation and stress induced cell death, although how they regulate these processes is unclear. Depletion of Hsp70 proteins tends to sensitize cells to apoptotic stimuli and conversely their over-expression lends protection from stress induced apoptosis(Saleh et al., 2000; Stankiewicz et al., 2005). Hsp70/40 proteins can also interact with and sequester Apaf1, a component of the cell death machinery (Saleh et al., 2000). While in the NSM, DNJ-11 has been shown to function upstream of CES-1, it remains to be determined if it also has a CES-1 independent function in regulating the Apaf1 homolog, CED-4. Since Snail homologs regulate ACD in C. elegans and Drosophila, an interesting question is whether DNJ-11 homologs also regulate ACD in organisms other than C. elegans.

\section{Why generate a cell only to kill it?}

Why would a cell expend energy to divide when one of its daughters dies? This puzzling phenomenon is particularly dramatic in nematodes, with the C. elegans hermphrodite producing 1090 cells and losing 131 to apoptosis. The ability to regulate apoptosis can make sense. Developmental apoptosis has been cited as a sculpting and culling mechanism, particularly in vertebrates(Meier et al., 2000) or during insect metamorphosis(Truman et al., 1992; Yin and Thummel, 2004). It has also been suggested that ridding excessive cells is often less risky than not having enough healthy cells(Meier et al., 2000). In nematodes, apoptosis can make sense in certain lineages. For example, a particular lineage that is reiterated at different places in the worm can differ by the presence or absence of a cell death(Sulston, 1976), and this regulation produces cells only in places where they are needed. There are also cases where a cell survives only in the sex where it is needed(Conradt and Horvitz, 1999).

For most apoptotic events in C. elegans, however, why cells are fated to die is unclear. One interesting hypothesis proposes that apoptotic cells can drift functionally, allowing them to acquire new traits that can be tested when the cell is allowed to survive(Avery and Horvitz, 1987). This idea is reminiscent of the "pseudogene hypothesis," as pseudogenes have no functional role in the organism, but may acquire novel functions by mutation. The "pseudocell hypothesis" is consistent with the observations that in ced mutants, when cells fated to die are allowed to survive, they have more variable fates than their siblings(Avery and Horvitz, 1987).

The finding that aggresomes are segregated during cell divisions suggests yet another model for the role of apoptosis in ACD. Perhaps in C. elegans, as in Drosophila neuroblasts, misfolded proteins segregate to cells that die. It seems unlikely that this segregation causes cell death since the same cells always die. If accumulated aggresomes triggered apoptosis, then conditions that favor aggresome formation would produce deaths earlier in the lineages and conditions that fail to produce aggresomes would allow the cells to survive. But the same cells always die. Instead, developmental apoptosis in some C.elegans lineages could provide repositories for misfolded proteins. We can envision two ways that apoptosis and aggresome segregation could have evolved. Some lineages could have originally segregated aggresomes to cells that served as repositories for misfolded proteins, protecting other cells in the lineage from their 
deleterious effects. Later, the apoptotic pathway could have been implemented to remove the nonfunctional cells. It is possible that initially, apoptosis was triggered by aggresome accumulation, but more reproducible mechanisms of turning on the apoptotic pathway would eventually have to evolve to ensure that the cells always died. It is also possible that these cells first died and the segregation of aggresomes to the apoptotic cells evolved later. We favor the former scenario because it provides an explanation for why specific cells in a lineage die.

While this model is not supported directly by any experimental evidence, three observations are consistent with it. First, cells inheriting aggregated proteins have a tendency to die. This apoptosis occurs in cultured cells and in Drosophila where the neuroblast dies after producing its repertoire of GMCs(Betschinger and Knoblich, 2004; Maurange and Gould, 2005). As with apoptosis in C. elegans, the deaths in Drosophila are programmed to occur after all of the GMCs are produced, suggesting that while aggresome accumulation might have initially triggered apoptosis, more reproducible mechanisms evolved to ensure the cell dies. Second, C. elegans DNJ-11 contains an Hsp40 domain, and Hsp40-domain containing proteins are found in aggresomes(Garcia-Mata et al., 2002). Levels of Hsp70/40 chaperones have been linked independently to both apoptotic fate and the formation of protein aggregates(Fortun et al., 2007; Saleh et al., 2000; Stankiewicz et al., 2005). Third, aggresomes have been suggested to act as cytoprotective recruitment centers to facilitate degradation of toxic proteins(Taylor et al., 2003). This model makes two predictions: (i) the apoptotic daughter will inherit the mother centriole, and (ii) the apoptotic daughter will inherit aggresomes in C.elegans lineages.

\section{In conclusion}

Coupling aggresomes to centrosomes may be a mechanism for dividing cells to maintain healthy cells at times when demands for new protein synthesis is high. This coupling could be a mechanism to keep longer lived or critical populations of cells, such as post-mitotic neurons or adult stem cells, free from the cellular damage that might result from extensive protein misfolding. It has been suggested that neurons are particularly susceptible to aggresomeinduced diseases since they are post-mitotic and cannot remove their aggresomes through rounds of cell divisions with subsequent apoptosis(Fuentealba et al., 2008). In cultured cells and Drosophila neuroblasts, cells that inherit aggregated proteins often die, their death either specified by a developmental program or triggered by an excess of aggregated proteins. How these cells decide when to die and how these decisions are transmitted to the apoptotic pathway are interesting questions that await answers. In Drosophila neuroblasts, it seems equally likely that after producing its repertoire of GMCs, the neuroblast is programmed to die independent of the amount of protein aggregates that have formed. We speculate that apoptosis in $C$. elegans may also provide a way of removing cells that contain misfolded proteins from the animal, the apoptotic cell near the end of the lineage serving as an incidental garbage can for any mis-folded protein aggresomes that may have formed. If so, it leaves us to wonder whether similar mechanisms could also operate during vertebrate development and ageing.

\section{Acknowledgments}

We apologize to any of our colleagues that we have failed to cite because of page constraints. This work was supported by National Institutes of Health grant NS42213 to G.G.

\section{References}

Acebron SP, et al. DnaJ recruits DnaK to protein aggregates. J Biol Chem 2008;283:1381-90. [PubMed: 17984091]

Ashraf SI, Ip YT. The Snail protein family regulates neuroblast expression of inscuteable and string, genes involved in asymmetry and cell division in Drosophila. Development 2001;128:4757-67. [PubMed: 11731456] 
Avery L, Horvitz HR. A cell that dies during wild-type C. elegans development can function as a neuron in a ced-3 mutant. Cell 1987;51:1071-8. [PubMed: 3690660]

Betschinger J, Knoblich JA. Dare to be different: asymmetric cell division in Drosophila, C. elegans and vertebrates. Curr Biol 2004;14:R674-85. [PubMed: 15324689]

Cai Y, et al. A family of snail-related zinc finger proteins regulates two distinct and parallel mechanisms that mediate Drosophila neuroblast asymmetric divisions. Embo J 2001;20:1704-14. [PubMed: 11285234]

Chalfie M, et al. Mutations that lead to reiterations in the cell lineages of C. elegans. Cell 1981;24:5969. [PubMed: 7237544]

Conradt B, Horvitz HR. The TRA-1A sex determination protein of C. elegans regulates sexually dimorphic cell deaths by repressing the egl-1 cell death activator gene. Cell 1999;98:317-27. [PubMed: 10458607]

Cordes S, et al. The C. elegans MELK ortholog PIG-1 regulates cell size asymmetry and daughter cell fate in asymmetric neuroblast divisions. Development 2006;133:2747-56. [PubMed: 16774992]

Delattre M, Gonczy P. The arithmetic of centrosome biogenesis. J Cell Sci 2004;117:1619-30. [PubMed: 15075224]

Ellis RE, Horvitz HR. Two C. elegans genes control the programmed deaths of specific cells in the pharynx. Development 1991;112:591-603. [PubMed: 1794327]

Fortun J, et al. The formation of peripheral myelin protein 22 aggregates is hindered by the enhancement of autophagy and expression of cytoplasmic chaperones. Neurobiol Dis 2007;25:252-65. [PubMed: 17174099]

Frank CA, et al. C. elegans HAM-1 positions the cleavage plane and regulates apoptosis in asymmetric neuroblast divisions. Dev Biol 2005;284:301-10. [PubMed: 15979607]

Frydman J, et al. Folding of nascent polypeptide chains in a high molecular mass assembly with molecular chaperones. Nature 1994;370:111-7. [PubMed: 8022479]

Fuentealba LC, et al. Asymmetric mitosis: Unequal segregation of proteins destined for degradation. Proc Natl Acad Sci U S A 2008;105:7732-7. [PubMed: 18511557]

Garcia-Mata R, et al. Hassles with taking out the garbage: aggravating aggresomes. Traffic 2002;3:38896. [PubMed: 12010457]

Goldstein B, Macara IG. The PAR proteins: fundamental players in animal cell polarization. Dev Cell 2007;13:609-22. [PubMed: 17981131]

Hatzold J, Conradt B. Control of apoptosis by asymmetric cell division. PLoS Biol 2008;6:e84. [PubMed: 18399720]

Horvitz HR, Herskowitz I. Mechanisms of asymmetric cell division: two Bs or not two Bs, that is the question. Cell 1992;68:237-55. [PubMed: 1733500]

Hundley HA, et al. Human Mpp11 J protein: ribosome-tethered molecular chaperones are ubiquitous. Science 2005;308:1032-4. [PubMed: 15802566]

Hut HM, et al. Hsp70 protects mitotic cells against heat-induced centrosome damage and division abnormalities. Mol Biol Cell 2005;16:3776-85. [PubMed: 15930131]

Jan YN, Jan LY. Asymmetric cell division in the Drosophila nervous system. Nat Rev Neurosci 2001;2:772-9. [PubMed: 11715054]

Johnston JA, et al. Cytoplasmic dynein/dynactin mediates the assembly of aggresomes. Cell Motil Cytoskeleton 2002;53:26-38. [PubMed: 12211113]

Johnston JA, et al. Aggresomes: a cellular response to misfolded proteins. J Cell Biol 1998;143:188398. [PubMed: 9864362]

Knoblich JA. Asymmetric cell division during animal development. Nat Rev Mol Cell Biol 2001;2:1120. [PubMed: 11413461]

Kopito RR. Aggresomes, inclusion bodies and protein aggregation. Trends Cell Biol 2000;10:524-30. [PubMed: 11121744]

Lundell MJ, et al. The regulation of apoptosis by Numb/Notch signaling in the serotonin lineage of Drosophila. Development 2003;130:4109-21. [PubMed: 12874131]

Maurange C, Gould AP. Brainy but not too brainy: starting and stopping neuroblast divisions in Drosophila. Trends Neurosci 2005;28:30-6. [PubMed: 15626494] 
Meier P, et al. Apoptosis in development. Nature 2000;407:796-801. [PubMed: 11048731]

Metzstein MM, et al. Transcriptional regulator of programmed cell death encoded by Caenorhabditis elegans gene ces-2. Nature 1996;382:545-7. [PubMed: 8700229]

Metzstein MM, Horvitz HR. The C. elegans cell death specification gene ces- 1 encodes a snail family zinc finger protein. Mol Cell 1999;4:309-19. [PubMed: 10518212]

Mizumoto K, Sawa H. Two betas or not two betas: regulation of asymmetric division by beta-catenin. Trends Cell Biol 2007;17:465-73. [PubMed: 17919911]

Otto H, et al. The chaperones MPP11 and Hsp70L1 form the mammalian ribosome-associated complex. Proc Natl Acad Sci U S A 2005;102:10064-9. [PubMed: 16002468]

Rebollo E, et al. Functionally unequal centrosomes drive spindle orientation in asymmetrically dividing Drosophila neural stem cells. Dev Cell 2007;12:467-74. [PubMed: 17336911]

Rujano MA, et al. Polarised asymmetric inheritance of accumulated protein damage in higher eukaryotes. PLoS Biol 2006;4:e417. [PubMed: 17147470]

Rusan NM, Peifer M. A role for a novel centrosome cycle in asymmetric cell division. J Cell Biol 2007;177:13-20. [PubMed: 17403931]

Saleh A, et al. Negative regulation of the Apaf-1 apoptosome by Hsp70. Nat Cell Biol 2000;2:476-83. [PubMed: 10934467]

Stankiewicz AR, et al. Hsp70 inhibits heat-induced apoptosis upstream of mitochondria by preventing Bax translocation. J Biol Chem 2005;280:38729-39. [PubMed: 16172114]

Stearns T. Centrosome duplication. a centriolar pas de deux. Cell 2001;105:417-20. [PubMed: 11371338]

Sulston JE. Post-embryonic development in the ventral cord of Caenorhabditis elegans. Philos Trans R Soc Lond B Biol Sci 1976;275:287-97. [PubMed: 8804]

Taylor JP, et al. Aggresomes protect cells by enhancing the degradation of toxic polyglutamine-containing protein. Hum Mol Genet 2003;12:749-57. [PubMed: 12651870]

Thellmann M, et al. The Snail-like CES-1 protein of C. elegans can block the expression of the BH3only cell-death activator gene egl-1 by antagonizing the function of bHLH proteins. Development 2003;130:4057-71. [PubMed: 12874127]

Truman JW, et al. Programmed neuronal death in insect development. J Neurobiol 1992;23:1295-311. [PubMed: 1469389]

Urbani L, Stearns T. The centrosome. Curr Biol 1999;9:R315-7. [PubMed: 10322119]

Vidair CA, et al. Heat shock alters centrosome organization leading to mitotic dysfunction and cell death. J Cell Physiol 1993;154:443-55. [PubMed: 8436595]

Wu WS, et al. Slug antagonizes p53-mediated apoptosis of hematopoietic progenitors by repressing puma. Cell 2005;123:641-53. [PubMed: 16286009]

Yamashita YM, et al. Asymmetric inheritance of mother versus daughter centrosome in stem cell division. Science 2007;315:518-21. [PubMed: 17255513]

Yin VP, Thummel CS. A balance between the diap1 death inhibitor and reaper and hid death inducers controls steroid-triggered cell death in Drosophila . Proc Natl Acad Sci U S A 2004;101:8022-7. [PubMed: 15150408] 

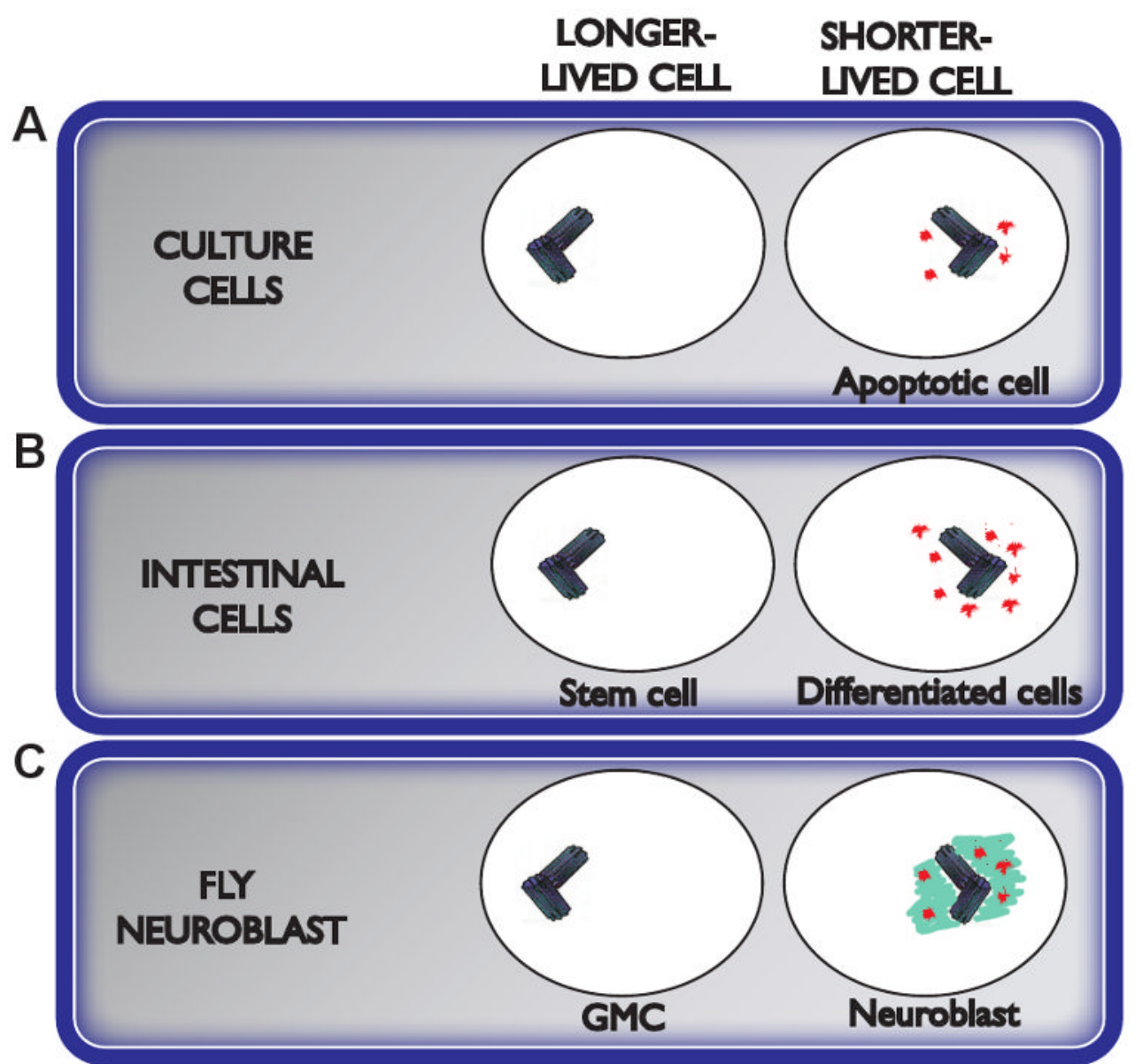

D

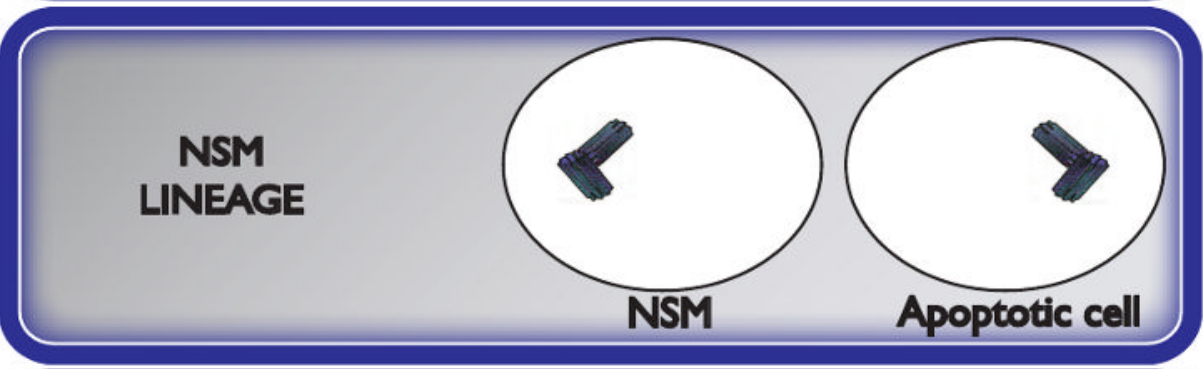

\section{Aggrosomes Centrioles Peri-centriolar material}

Figure 1.

A comparison of a cell's "shelf-life," PCM inheritance, aggresome inheritance and apoptotic fate during asymmetric divisions. Daughter cells from each ACD are aligned vertically as "shorter-" and "longer-" lived cells. The lowermost panel defines the cartoon depictions. (A) Fly neuroblasts are shorter lived, compared to GMC derivatives. Embryonic neuroblasts inherit aggresomes, and larval neuroblasts inherit the mother centrosome/PCM(Rebollo et al., 2007; Rujano et al., 2006; Rusan and Peifer, 2007). (B) Aggresomes associate with the PCM, and in cultured cells, segregate to the daughter with a higher apoptotic fate potential(Rujano et al., 2006). (C) Aggresomes are prevalent in the shorter-lived differentiated cells of the human intestinal crypt(Rujano et al., 2006).(D) NSM neuroblast divisions generate an apoptotic 
daughter. The inheritance pattern of the mother centrosome/PCM and aggresomes are unknown. 

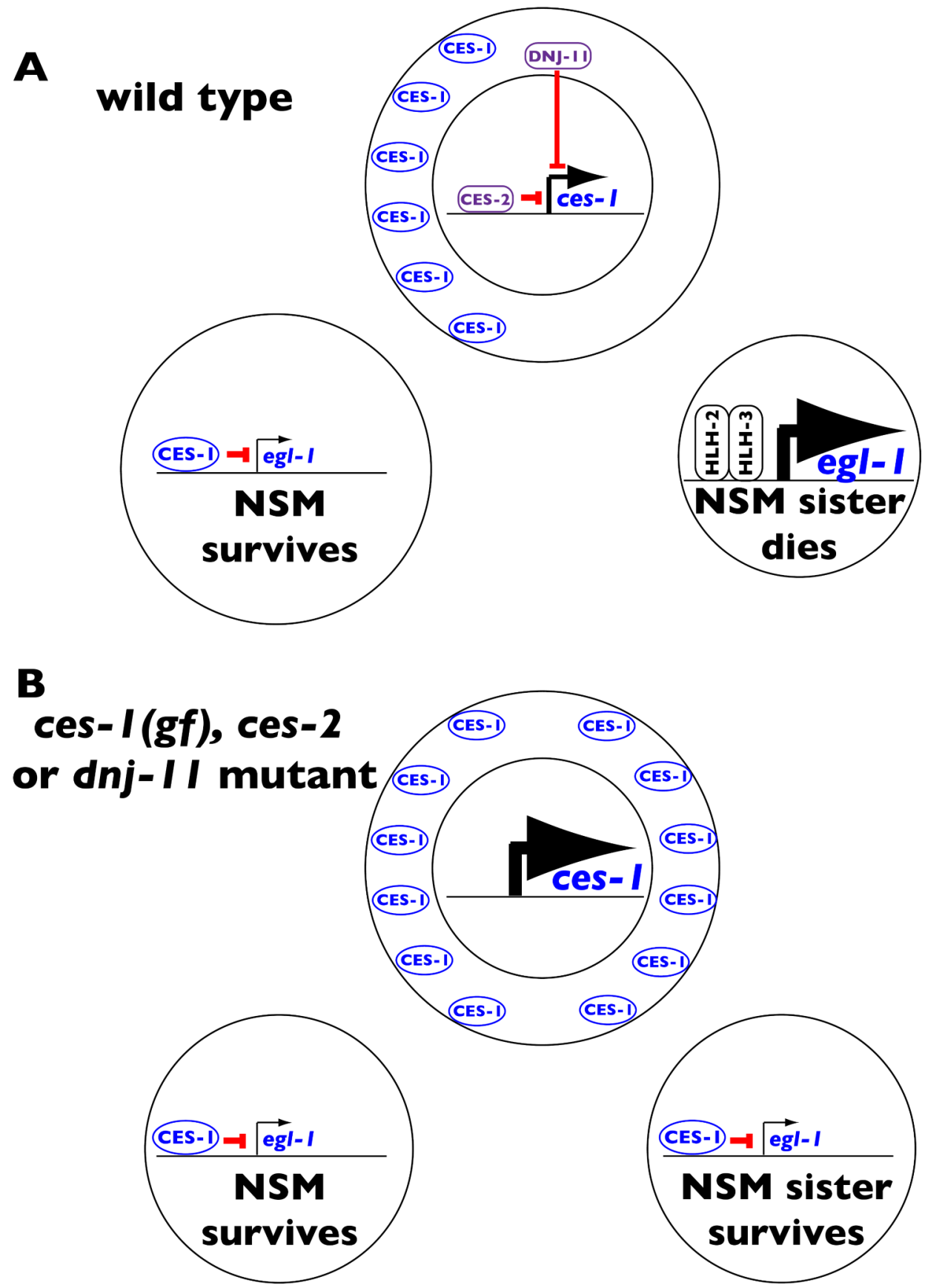

Figure 2.

Model for the division of the NSM neuroblast. In the wild-type lineage (top), the gene ces- 1 is inhibited by CES-2 and DNJ-11. How DNJ-11 inhibits ces-1 is unclear. CES-1 protein is asymmetrically distributed to the NSM, where it inhibits binding of the HLH-2/HLH-3 heterodimer to sequences that regulate the proapoptotic gene egl-1, preventing expression expression of egl-1. Lack of CES-1 protein in the NSM sister allows HLH-2/HLH-3 to promote egl-l expression, which leads to apoptosis. (Bottom) A gain-of-function mutation in ces- 1 or loss-of-function mutations in either ces-2 or $d n j-11$ lead to excess expression of ces- 1 , causing the daughter cells to become more equivalent in size and the NSM sister to survive. Adapted from Hatzold and Conradt(Hatzold and Conradt, 2008). 